\title{
Multi-Mode lot Gateway Design and Implemention
}

\author{
Pengcheng Sun ${ }^{1}$, a, Dong $\mathrm{Li}^{2,}$ b \\ ${ }^{1}$ School of Automation, Wuhan University of Technology, Wuhan 430000, China; \\ ${ }^{2}$ School of Computer Science and Technology, Wuhan University of Technology, Wuhan 430000, \\ China. \\ aslanpo@163.com, bLidongxy@163.com
}

Keywords: Heterogenous, loT Gateway, Data Processing.

\begin{abstract}
In this paper, a method for the design and implementation of the Internet of Things (IoT) gateway is proposed. First, the gateway platform is built on BeagleBone Black. In the software design of the gateway, wireless sensor network mapping into a virtual device through the Lua scripting language, and then through the operation of virtual equipment to achieve the dynamic processing of heterogeneous data and access to heterogeneous network, finally converts the heterogeneous data into JSON format data for cloud platform.
\end{abstract}

\section{Introduction}

With the rapid development of IoT, sensors can access to net by WIFI, BLE, Zigbee or Sub1GHz and so on. For different kinds of access method, it is necessary to realize interconnection among heterogeneous networks, cooperation among various devices and utilization and management of kinds of heterogeneous resources. As a link between IoT sensing layer and traditional communication network, IoT gateway is a key device for IoT application while protocol conversion between sensing layer and fundamental network [1]. It is important to realize a kind of gateway which has dynamic probability for heterogeneous devices access and data processing. In view of this, a new method for IoT gateway design and implementation is proposed for multisource heterogeneous data processing.

\section{Overall Design}

Gateway is usually based on an embedded platform on hardware. It processes data of corresponding sensor network through different modules, and then communicates with Ethernet and mobile wireless network through external network interface. The framework is shown in Figure 1.

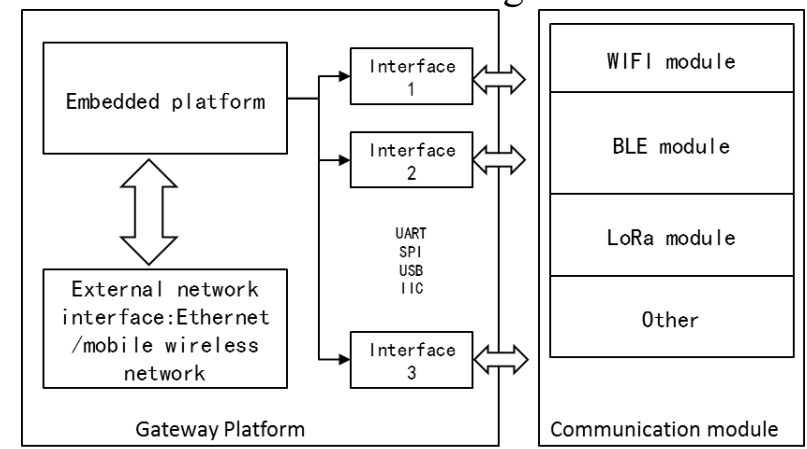

Fig. 1 The framework of the gateway

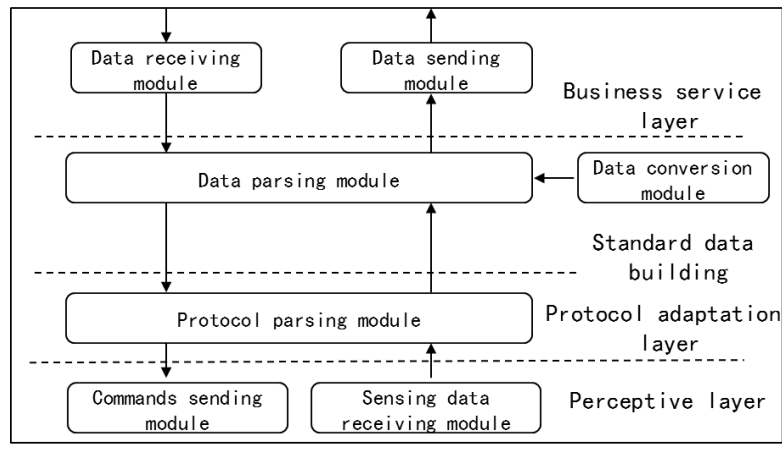

Fig. 2 The software module of the gateway

The software module of the gateway is shown in Figure 2.

1. The perceptual communication module is responsible for communicating with different networks.

2. The protocol adaptation module is responsible for the identification and analysis of the collected data.

3. The data conversion module is responsible for converting it into standardized data.

4. The business service module is responsible for transferring the data to the remote server. 


\section{Build Platform}

For gateway design, it is very important to choose a suitable embedded software and hardware platform. The platform must provide enough interfaces, such as UART, SOCKET, SPI, IIC and so on. At the same time, it also needs good interface performance and provides support for various modules. The processing of heterogeneous data is mainly dependent on the computing power of the gateway platform.

\subsection{Embedded Software and Hardware Platform.}

In this article, the hardware platform of the gateway uses the BeagleBone Black (BBB) designed by the Ti. As shown in Figure 3 ,It provides a rich hardware interface, it can work with various communication modules to meet different application needs, and most importantly, as an open source platform, it is very suitable for use as gateway of the Internet of things[2]. By running Linux on BBB, we access the Sub1GHz module and BLE module through UART interface, so that it has the function of accessing the corresponding wireless network, and access the cloud server through the Ethernet interface of the platform.

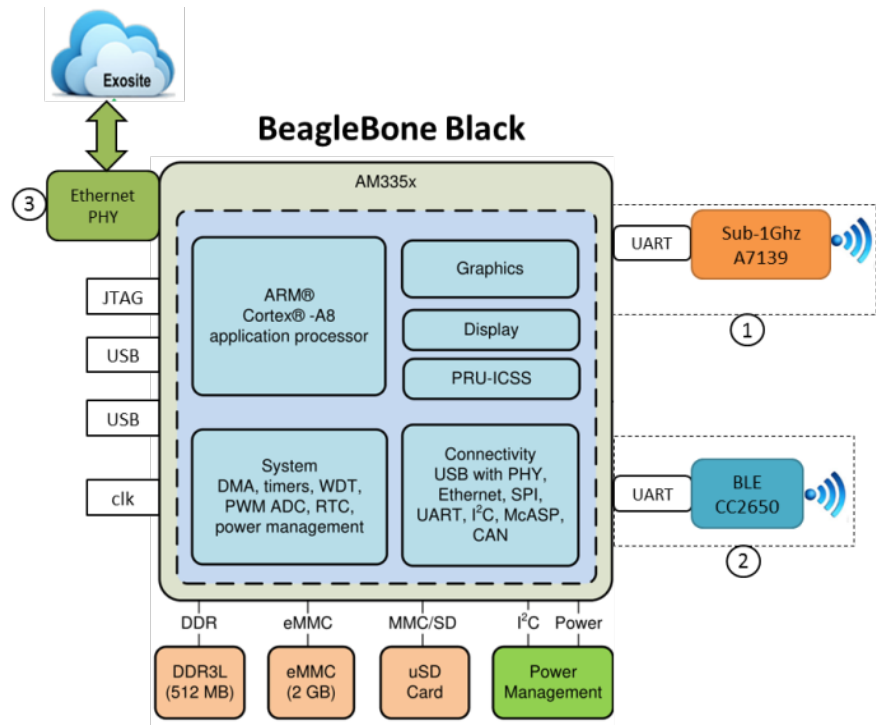

Fig. 3 Gateway platform

The communication module used above is A7139 and the CC2650. Although the principles of all kinds of front end communication modules are different, their interfaces with embedded platforms usually adopt UART, USB, SPI and so on. The design method of this paper is also applicable to other modules, which has strong representativeness and is easy to expand according to various needs.

\subsection{Communication Module.}

After selecting the platform, different modules can be selected to access different networks according to different usage requirements, as shown in the following table.

Table 1 Wireless communication module

\begin{tabular}{|c|c|c|c|c|}
\hline Variable & Wi-Fi & Z-Wave & ZigBee & Thread \\
\hline $\begin{array}{l}\text { Year first launched } \\
\text { in Market }\end{array}$ & 1997 & 2003 & 2003 & 2015 \\
\hline $\begin{array}{l}\text { PHY/MAC } \\
\text { Standard }\end{array}$ & $\begin{array}{c}\text { IEEE } \\
802.11 .1 \\
\end{array}$ & ITU-T G.9959 & $\begin{array}{c}\text { IEEE } \\
802.15 .4 \\
\end{array}$ & $\begin{array}{c}\text { IEEE } \\
802.15 .4 \\
\end{array}$ \\
\hline Frequency Band & $2.4 \mathrm{GHz}$ & $900 \mathrm{MHz}^{*}$ & $2.4 \mathrm{GHz}$ & $2.4 \mathrm{GHz}$ \\
\hline $\begin{array}{l}\text { Nominal Range } \\
(0 \mathrm{dBm})\end{array}$ & $100 \mathrm{~m}$ & $30-100 m$ & $10-100 m$ & $10-100$ \\
\hline $\begin{array}{l}\text { Maximum Data } \\
\text { Rate }\end{array}$ & $54 \mathrm{Mbit} / \mathrm{s}$ & $40-100 \mathrm{kbit} / \mathrm{s}$ & $250 \mathrm{kbit} / \mathrm{s}$ & $250 \mathrm{kbit} / \mathrm{s}$ \\
\hline Topology & Star & Mesh & Mesh & Mesh \\
\hline
\end{tabular}




\section{LUA-Based Programming}

In many cases, sensor data is changeable. The way of network access is changeable. The application demand of data is changeable. It is very difficult for gateway to flexibly access various networks, and intelligent analysis and processing of different data is aslo very difficult. It is difficult to develop such a gateway program in the traditional way, and the gateway program is not easy to expand.

Based on the Lua scripting language, this paper designs a group of universal processing programs that can be used for fast development gateway to match various heterogeneous networks and heterogeneous data. The characteristics of the dynamic scripting language are suitable for the diversity and variability of protocols and devices, and are easily changed according to the changes in the application.

By configure the access device operation model, device data model, device communication protocol and communication interface in the Lua script, and map the collection of these scripts to a virtual device. In the gateway's host program, according to the configuration of virtual devices, a Lua virtual machine is opened to process corresponding transactions.

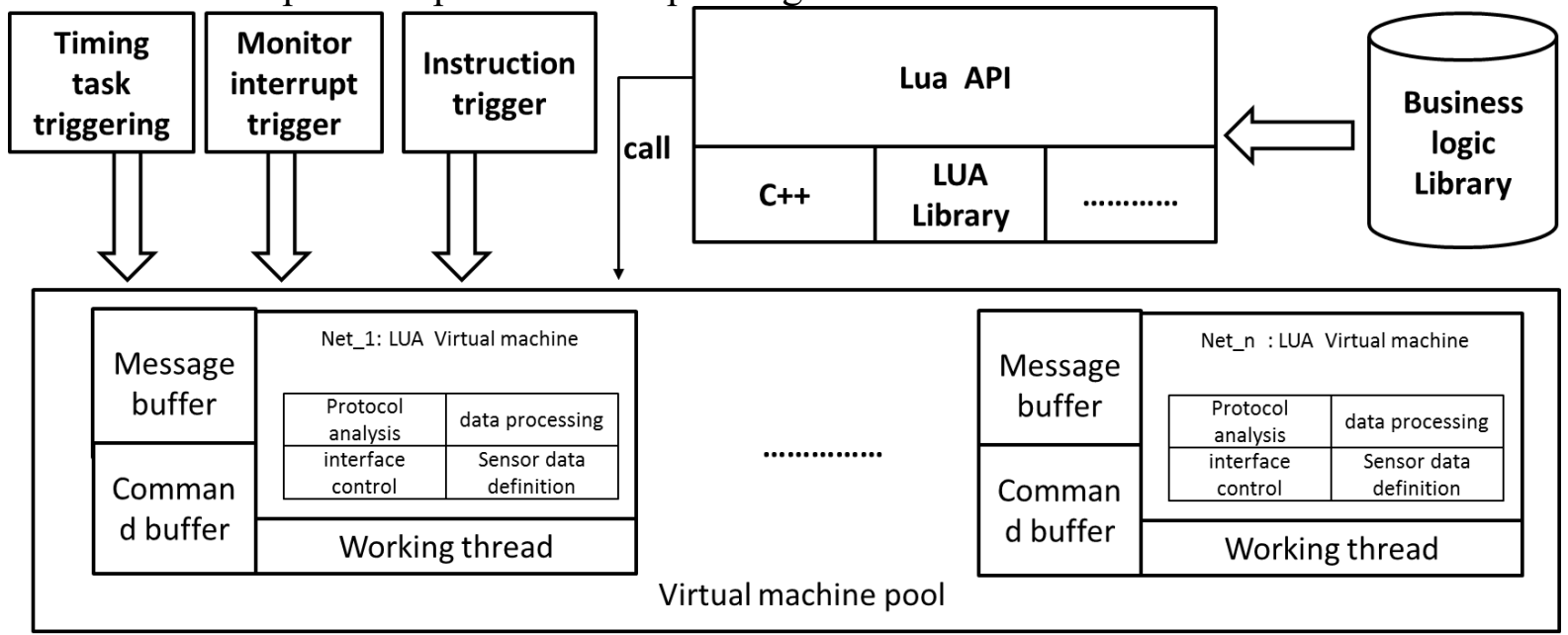

Fig. 4 General processing program

As shown in Figure 4, on the basis of the use of the Lua scripting language to define the virtual device,In this paper, a multi-thread operation mode is set up to extract data from multiple heterogeneous networks.Then, each heterogeneous sensing network module is analyzed independently in the adaptive layer of the protocol.Finally, the data on multiple perceptive networks can be effectively extracted.So each independent adaptation module corresponds to a thread, This way through multi- thread concurrent execution can realize the access to various heterogeneous sensing networks and the collection of heterogeneous data.

For new devices and protocols, only need to complete the data model definition and encoding program, this method simply by changing the parameters can fit all kinds of network access and processing business, the gateway program encoding is simpler and more stable operation of the gateway.

\section{Access to Heterogeneous Network}

The concurrent access of heterogeneous networks is the primary task of the gateway.The most difficult problems in multiple heterogeneous networks have been solved after starting a virtual device defined by the Lua language through multi-thread.Now, the access of multiple networks is properly configured and using the corresponding communication module through the program.The corresponding communication module is connected to the platform interface. What the program needs to deal with is the management and application of the corresponding interfaces. The SOCKET interface and UART interface management and application in this paper are shown in Figure 5. 

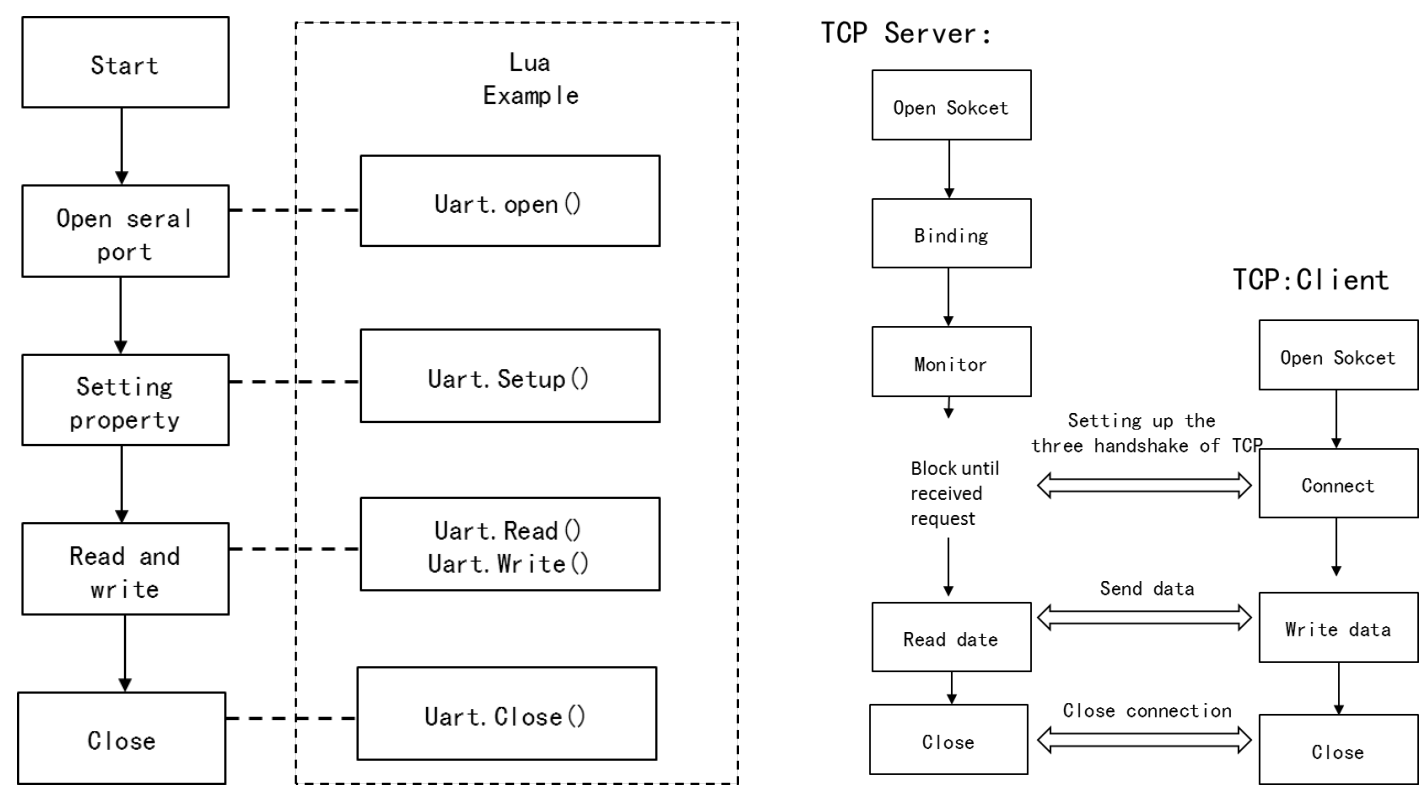

Fig. 5 Examples of the use of UART and SOCKET

\section{Processing Heterogeneous Data}

After the concurrent access to heterogeneous networks, the core work of the gateway is to receive and process data.In data transmitted from heterogeneous networks, there are a large number of invalid data, such as indiscriminate receiving and judging processing one by one, which will consume a large amount of system resources.In addition, all kinds of sensor information are used in different formats, and both interpretation and processing are corresponding to different rules.

\subsection{Protocol Adaptation.}

The protocol adaptation is implemented by the Lua protocol adaptation script.In addition to the main program of the gateway, independent Lua scripts are created to realize dynamic identification of different sensor protocols.According to the protocol adaptation rules pre - declared by Lua, different methods can be used for data processing.

When the corresponding virtual machine is opened, the corresponding protocol adaptation script is loaded. First, it can judge the integrity of the received data. Secondly, it can also analyze the data according to the pre-rule, extract effective data for further processing.

\subsection{Data Conversion.}

The main purpose of the gateway is to realize the transformation between different protocols. The gateway implemented in this paper designs a standard JSON format middleware protocol for various heterogeneous data in the process of processing. The program can be converted into the standard format, regardless of which message format is received.In order to realize the unification of different message formats and facilitate the conversion of message format to each other.In data exchange, the JSON data format is a lightweight "key/value" data exchange format.Compared with the common XML, JSON has less capacity in describing the same device, so that it can transmit the same data with less traffic in transmission.

For example, the following figure 6 is the process of temperature and humidity data predefined and processed through Lua scripts, and eventually converted into a JSON format. 

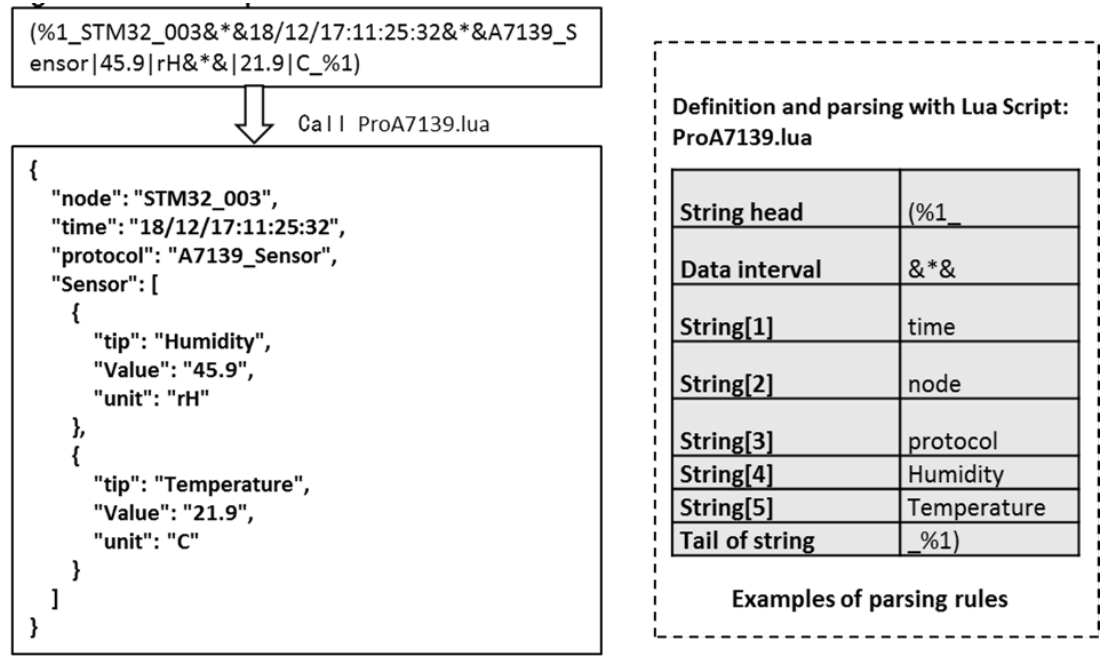

Fig. 6 Examples of standardization of data conversion

\section{Application Services for Cloud Platforms}

On the basis of the previous article, the JSON format data can be sent to the cloud platform through the IoT protocol, such as MQTT or COAP. Application layer can be quickly deployed based on cloud - based applications based on requirements.

In short, it is standardized through the gateway. The final JSON data format has a very wide range of support, such as:

1. Develop the Internet of things program based on the subscription and push mechanism [3].

2. Persisting the data, storing data in MongoDB and other databases in the form of documents to support the traditional database-based data application requirements [4].

3. Developing Web Applications [5].

\section{Conclusion}

The application shows that this method can effectively improve the development efficiency of the gateway of the Internet of things, and the gateway developed can be applied to multiple network conditions and multi-sensor access.

\section{References}

[1]. Zhu Q, Wang R, Chen Q, et al. IOT Gateway: BridgingWireless Sensor Networks into Internet of Things[C]// Ieee/ifip, International Conference on Embedded and Ubiquitous Computing. IEEE, 2011:347-352.

[2]. Information on: beagleboard.org.

[3]. Vargas D C Y, Salvador C E P. Smart IoT Gateway For Heterogeneous Devices Interoperability[J]. IEEE Latin America Transactions, 2016, 14(8):3900-3906.

[4]. Sellami R, Bhiri S, Defude B. Supporting Multi Data Stores Applications in Cloud Environments[J]. IEEE Transactions on Services Computing, 2016, 9(1):59-71.

[5]. Liu F, Chen P, Jia J Y. Application of WebSocket and MQTT in Web Real-Time Communication System[J]. Computer Systems \& Applications, 2016. 